\title{
A 68-year-old woman with recurrent cystitis
}

\author{
Monica Mutyala MD¹, Ruba A Halloush MD², Faisal A Khasawneh MD³
}

\section{CASE PRESENTATION}

A 68-year-old Hispanic woman presented with a third episode of cystitis. She complained of multiple episodes of dysuria, frequency and suprapubic discomfort for the past six months. She denied fevers, chills, hematuria and flank pain. There was no history of nephrolithiasis and she had no other complaints. Her symptoms responded to oral levofloxacin, administered for 14 days after each episode of infection; however, they recurred a few weeks thereafter. Her medical history was significant for diabetes mellitus that was well controlled on oral hypoglycemic agents. She did not smoke, had immigrated to the United States 45 years previously and had a negative purified protein derivative test on intake. She was married with three healthy children, was a homemaker and had no contact with animals.

On examination, the patient was afebrile, with a temperature of $37.6^{\circ} \mathrm{C}$. Her blood pressure was $126 / 78 \mathrm{mmHg}$, heart rate was 82 beats/min and her oxygen saturation was $97 \%$ on room air. She was awake, alert and not in acute distress. Her skin was intact with no rashes or lymphadenopathy. She had clear lung fields, regular heart sounds without murmurs and soft nontender abdomen with no organomegaly. She had no renal angle tenderness and no lymphadenopathy. Her neurological examination was nonfocal.

Blood tests revealed a hemoglobin level of $97 \mathrm{~g} / \mathrm{L}$ and white blood cell count of $8.4 \times 10^{9}$ cells/L. She had chronic kidney disease with a creatinine level of $133 \mu \mathrm{mol} / \mathrm{L}$. Her liver function tests were normal. Urinalysis revealed a white blood cell count of $>100$ cells per highpower field, with hematuria and proteinuria. Routine urine culture was negative. A computed tomography scan of the abdomen and pelvis showed normal kidney size with no stones, and a thickened urinary bladder wall with no ascites or lymphadenopathy. The patient underwent cystoscopy and biopsy (Figures 1 and 2).

What is your diagnosis?

\section{DIAGNOSIS}

The patient's mucosal biopsy showed multiple granulomas composed of epithelioid histiocytes and multinucleated giant cells with acid-fast bacilli consistent with Mycobacterium tuberculosis (MTB). She did not have symptoms or radiological features concerning for pulmonary tuberculosis (TB). She was diagnosed with TB cystitis and started empirically on isoniazid (INH), rifampin (RIF), ethambutol and pyrazinamide. Biopsy culture confirmed the diagnosis and grew susceptible MTB. She completed two months of all four agents and a subsequent four months of INH and RIF. She tolerated the medications well, with complete resolution of her symptoms. The fact that levofloxacin is a second-line agent for treating TB explained the short-lived improvement in the patient's symptoms after the first two episodes of cystitis.

\section{DISCUSSION}

TB is encountered globally and is associated with high morbidity and mortality (1-3). Only $10 \%$ of TB-infected individuals will develop active disease $(4,5)$. The remainder have latent TB infection, which will continue to be dormant for their entire lives. Immunosuppression due to
HIV infection, long-term steroid use or organ transplantation, diabetes mellitus and chronic kidney disease can lead to TB reactivation $(5,6)$.

Genitourinary tuberculosis (GUTB) is a form of extrapulmonary TB and usually results from reactivation of a latent TB infection (7). The genitourinary tract is the second most common site of extrapulmonary $\mathrm{TB}$, following the lymphatic system $(4,6)$. The kidneys are the most common location for GUTB and are infected through hematogenous seeding. From the kidneys, the bacilli may spread through the urinary tract and nearby structures, which can cause infections in the retroperitoneum, adrenals, urinary collecting system, genital organs in men and pelvic organs in women $(6,7)$. Of note, isolated renal TB is more likely to affect young women in their third decade of life $(8,9)$.

In Canada, the rates of TB have been declining since 1970 (10). GUTB prevalence, however, has been stable (approximately 2\%) (11). In 2001, for example, GUTB accounted for $1.8 \%$ of all TB cases and $6.5 \%$ of extrapulmonary TB cases (12).

Patients with GUTB can present with symptoms similar to bacterial urinary tract infection (UTI). The most common presenting symptoms include irritative voiding symptoms, flank pain and hematuria (13). Additionally, systemic symptoms, such as fever and weight loss, may be present but are typically rare. Some patients can be asymptomatic; in these cases, pyuria and/or microscopic hematuria may be evident (14). In a retrospective study, Kulchavenya et al (8) found that the majority of patients with GUTB complained of flank pain $(35.2 \%)$ and dysuria (39.8\%). Similarly, in a 13-year study, Singh et al (9) observed that the most common clinical presentation of GUTB was irritative voiding symptoms $(66.47 \%)$.

The diagnosis of GUTB is based on the presence of TB risk factors and a long-standing history of urinary tract complaints that are often nonspecific, in addition to isolating MTB in urine or urinary tract tissue biopsy $(7,15)$. In the appropriate setting, pyuria in the absence of bacterial growth on routine microbiological studies should raise concerns for an underlying GUTB $(6,7)$.

Isolating MTB in urine has been used as the primary test in the diagnosis of GUTB (16). The sensitivity of urine culture in conventional culture media has been reported to be between $80 \%$ to $97 \%$ (15). Recently, nucleic acid amplification techniques, such as polymerase chain reaction (PCR), have been used more commonly for the detection of MTB (12). PCR is highly sensitive and specific in detecting MTB in urine, with reported sensitivity and specificity of up to $88 \%$ and $95 \%$, respectively $(4,15)$. Singh et al $(9)$ noted that PCR testing had a higher positivity rate $(67.7 \%)$ when compared with urine acid-fast bacillus staining (41.6\%) and urine MTB culture (55.4\%).

The purified protein derivative skin test has been traditionally used to evaluate previous exposure to TB. A positive skin test can support the diagnosis of TB; however, a negative skin test does not rule out pulmonary or extrapulmonary TB such as GUTB (17). Interferon-gamma release assays are also used for diagnosing latent tuberculosis infection. The specificity is $>95 \%$ and the sensitivity is $80 \%$ to $90 \%(18,19)$; however, there are limited data regarding the utility of interferon-gamma release assays for diagnosing GUTB (20).

${ }^{1}$ Department of Internal Medicine, Texas Tech University Health Sciences Center; ${ }^{2}$ Amarillo Pathology Group; ${ }^{3}$ Section of Infectious Diseases, Department of Internal Medicine, Texas Tech University Health Sciences Center, Amarillo, Texas, USA

Correspondence: Dr Faisal A Khasawneh, Section of Infectious Diseases, Department of Internal Medicine, Texas Tech University Health Science Center, 1400 South Coulter Street, Amarillo, Texas 79106, USA. TelepOhone 806-414-9110, fax 806-354-5764, email: faisal.khasawneh@ttuhsc.edu 


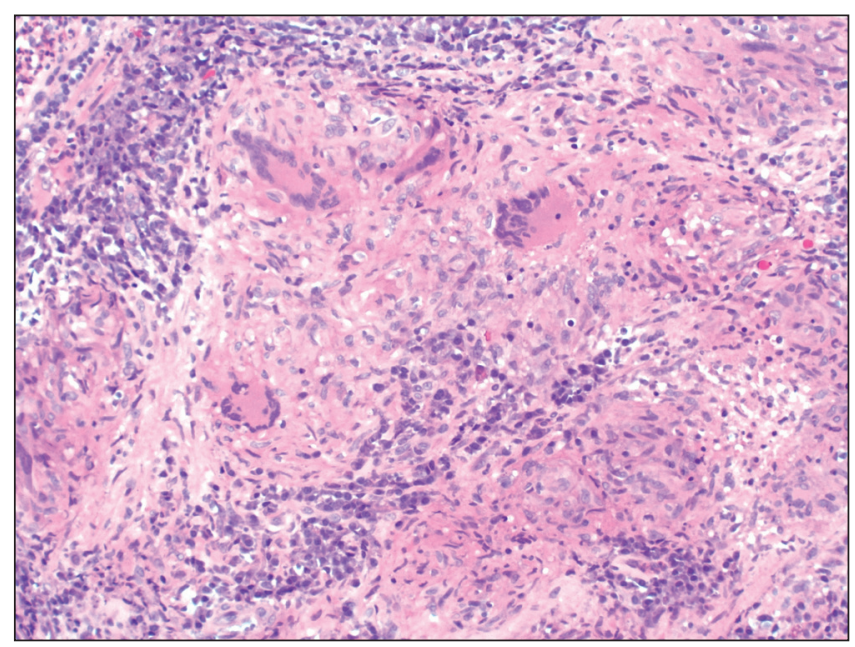

Figure 1) Hematoxylin and eosin-stained histological section of the bladder biopsy showing multiple granulomas composed of epithelioid histiocytes and multinucleated giant cells. Original magnification $\times 400$

Radiographic studies, including intravenous urography (IVU), plain abdominal radiography, ultrasonography, computed tomography and magnetic resonance imaging, can provide important clues in the diagnosis of GUTB. IVU was considered to be the gold-standard test in the diagnosis of early GUTB because it provides anatomical as well as functional details of the kidneys $(1,15)$. IVU can show calyceal erosions (a 'moth-eaten' appearance), which later progresses to papillary necrosis (21). Plain abdominal radiography can show renal calcification; however, this is only observed in $24 \%$ to $44 \%$ of GUTB cases (22). Focal and diffuse areas of lower echogenicity can be evident on kidney ultrasonography (21). Other features of GUTB observed on ultrasonography include calcifications, urothelial thickening, parenchymal masses and cavities (1). Computed tomography, however, is considered to be more sensitive than ultrasonography for the diagnosis of GUTB $(21,23)$, and can also help in evaluating renal function. Magnetic resonance imaging provides excellent details of kidney morphology and allows for the visualization of ureters (15). It has been used in challenging cases in which computed tomography and ultrasound images were inconclusive (24).

GUTB treatment is similar to that of pulmonary TB. It consists of the four first-line anti-TB drugs (INH, RIF, pyrazinamide and ethambutol) given for two months followed by INH and RIF given for four more months $(25,26)$. INH and RIF are considered to be the most active drugs in the regimen $(27,28)$. Second-line TB drugs, such as fluoroquinolones (FQs), are considered when the infection is caused by drug-resistant MTB, when the patient fails to achieve clinical response or when the patient experiences toxicity attributed to the standard treatment regimen $(3,28)$.

Bacterial UTI and GUTB can present with similar symptoms and signs (12). The initial empirical treatment of a complicated bacterial

\section{REFERENCES}

1. Merchant S, Bharati A, Merchant N. Tuberculosis of the genitourinary system - urinary tract tuberculosis: Renal tuberculosis - Part I. Indian J Radiol Imaging 2013;23:46-63.

2. Merchant S, Bharati A, Merchant N. Tuberculosis of the genitourinary system - urinary tract tuberculosis: Renal tuberculosis - Part II. Indian J Radiol Imaging 2013;23:64-77.

3. Fauci AS. Multidrug-resistant and extensively drug-resistant tuberculosis: The National Institute of Allergy and Infectious Diseases research agenda and recommendations for priority research. J Infect Dis 2008;197:1493-98.

4. Daher Ede F, da Silva GB Jr, Barros EJ. Renal tuberculosis in the modern era. Am J Tropical Medicine Hygiene 2013;88:54-64.

5. Ahmad S, Mokaddas E. Current status and future trends in the diagnosis and treatment of drug-susceptible and multidrug-resistant tuberculosis. J Infect Public Health 2014;7:75-91

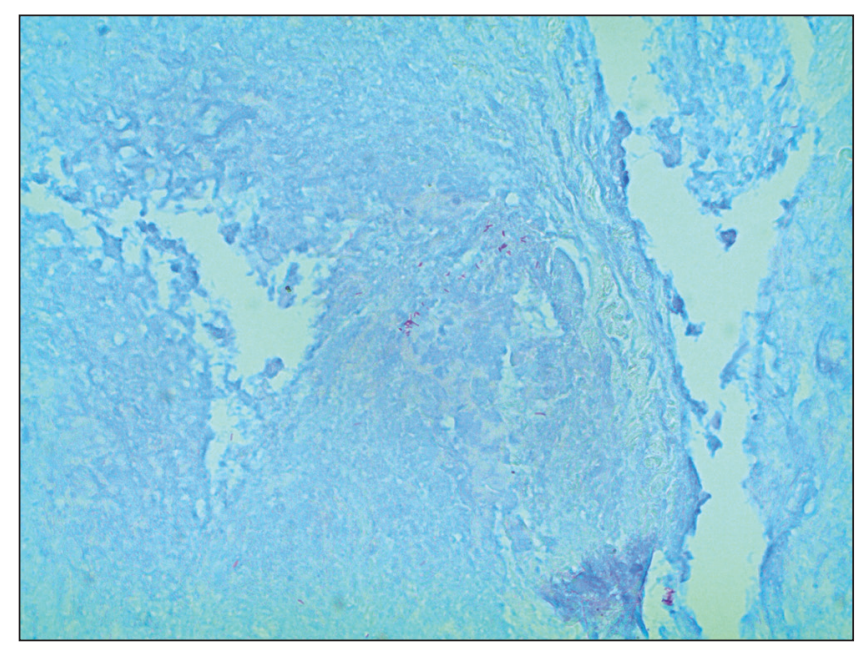

Figure 2) Acid-fast bacilli special stain showing clusters of mycobacteria (stained red). Original magnification $\times 400$

UTI may include FQs. In a study conducted in Alberta, Webster et al (10) observed that FQ monotherapy of unsuspected GUTB, as occurred in the present case, may delay diagnosis and lead to FQ resistance. In this retrospective study of culture-proven GUTB, three of 74 (4\%) MTB isolates were resistant to FQs. FQ-exposed isolates were more likely to be FQ resistant than nonexposed isolates (22\% versus $1.5 \% ; \mathrm{P}=0.04)$. Furthermore, among 41 cases $(55.4 \%)$ with symptoms and signs of UTI, eight $(19.5 \%)$ received a FQ, seven $(87.5 \%)$ of whom had delayed GUTB diagnosis.

Although anti-TB drugs are the first-line therapy for GUTB, surgical intervention may be indicated in certain cases such as abscess formation, nonfunctional kidneys caused by a late TB diagnosis, ureteric obstruction, and advanced unilateral disease complicated by pain or hemorrhage for which medical therapy has failed $(14,18)$. The need for GUTB surgical management has been declining, with a reported incidence of approximately $0.5 \%$ of all urological procedures (17). Unless used to drain an abscess or relieve obstructive uropathy, surgical intervention should not be considered until medical therapy has been provided for at least four to six weeks (29).

\section{CONCLUSION}

GUTB is the second most common form of extrapulmonary TB. Its symptoms and signs are nonspecific and can mimic those of bacterial UTI. Sterile puyria and recurrent UTI that is poorly responsive to antibiotic therapy should raise suspicion for this mycobacterial infection. Giving FQs in GUTB misdiagnosed as bacterial UTI can delay TB diagnosis and may lead to mycobacterial FQ resistance.

DISCLOSURES: The authors have no conflicts of interest to declare.

6. Wise GJ, Marella VK. Genitourinary manifestations of tuberculosis. Urol Clin North Am 2003;30:111-21.

7. Zajaczkowski T. Genitourinary tuberculosis: Historical and basic science review: Past and present. Cent European J Urol 2012;65:182-7.

8. Kulchavenya E, Zhukova I, Kholtobin D. Spectrum of urogenital tuberculosis. J Infect Chemother 2013;19:880-3.

9. Singh JP, Priyadarshi V, Kundu AK, Vijay MK, Bera MK, Pal DK. Genito-urinary tuberculosis revisited - 13 years' experience of a single centre. Indian J Tuberc 2013;60:15-22.

10. Webster D, Long R, Shandro C, et al. Fluoroquinolone resistance in renal isolates of Mycobacterium tuberculosis. Int J Tuberc Lung Dis 2010;14:217-22.

11. Enarson DA, Ashley MJ, Grzybowski S, Ostpkowickz E, Dorken E. Non-respiratory tuberculosis in Canada. Am J Epidemiol 1980;112:341-51. 
12. Eastwood JB, Corbishley CM, Grange JM. Tuberculosis and the kidney. J Am Soc Nephrol 2001;12:1307-14.

13. Kim JK, Bang WJ, Oh CY, Yoo C, Cho JS. Feasibility of the interferon $-\gamma$ release assay for the diagnosis of genitourinary tuberculosis in an endemic area. Korean J Urol 2013;54:123-6.

14. Simon HB, Weinstein AJ, Pasternak MS, Swartz MN, Kunz LJ. Genitourinary tuberculosis. Clinical features in a general hospital population. Am J Med 1977;63:410-20.

15. Kapoor R, Ansari MS, Mandhani A, Gulia A. Clinical presentation and diagnostic approach in cases of genitourinary tuberculosis. Indian J Urol 2008;24:401-5.

16. Diagnostic Standards and Classification of Tuberculosis in Adults and Children. This official statement of the American Thoracic Society and the Centers for Disease Control and Prevention was adopted by the ATS Board of Directors, July 1999. This statement was endorsed by the Council of the Infectious Disease Society of America, September 1999. Am J Respir Crit Care Med 2000;161:1376-95.

17. Cek M, Lenk S, Naber KG. EAU guidelines for the management of genitourinary tuberculosis. Eur Urol 2005;48:353-62.

18. Menzies D, Pai M, Comstock G. Meta-analysis: New tests for the diagnosis of latent tuberculosis infection: Areas of uncertainty and recommendations for research. Ann Intern Med 2007;146:340-54.

19. Pai M, Zwerling A, Menzies D. Systematic review: T-cell-based assays for the diagnosis of latent tuberculosis infection: An update. Ann Intern Med 2008;149:177-84.
20. Feng Y, Diao N, Shao L, et al. Interferon-gamma release assay performance in pulmonary and extrapulmonary tuberculosis. PLoS One 2012;7:e32652.

21. Maclean KA, Becker AK, Chang SD, Harris AC. Extrapulmonary tuberculosis: Imaging features beyond the chest. Can Assoc Radiol J 2013;64:319-24.

22. Hartman DS. Radiologic, pathologic correlation of the granulomatous lesions of the kidney. In: Stamey TA, ed. Monographs in Urology. London: Burroughs Wellcome Company, 1985.

23. Lu P, Li C, Zhou X. [Significance of the CT scan in renal tuberculosis]. Zhonghua Jie He He Hu Xi Za Zhi 2001;24:407-9.

24. Verswijvel G, Oyen R. Magnetic resonance imaging in the detection and characterization of renal diseases. Saudi J Kidney Dis Transpl 2004;15:283-99.

25. Gow JG, Barbosa S. Genitourinary tuberculosis. A study of 1117 cases over a period of 34 years. Br J Urol 1984;56:449-55.

26. Kadhiravan T, Sharma SK. Medical management of genitourinary tuberculosis. Indian J Urol 2008;24:362-8.

27. Migliori GB, D’Arcy Richardson M, Sotgiu G, Lange C. Multidrug-resistant and extensively drug-resistant tuberculosis in the West. Europe and United States: Epidemiology, surveillance, and control. Clin Chest Med 2009;30:637-65.

28. Katzung BG, Masters SB, Trevor AJ. Basic and Clinical Pharmacology. 12th edn. New York: McGraw-Hill Medical Publishing, 2012;803-10.

29. A Rai, H Pahwa, V Jain, S Misra. Management of genito-urinary tuberculosis. Internet J Surg 2009;23(1). 


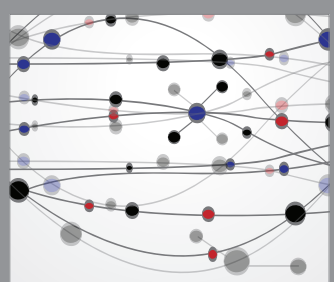

The Scientific World Journal
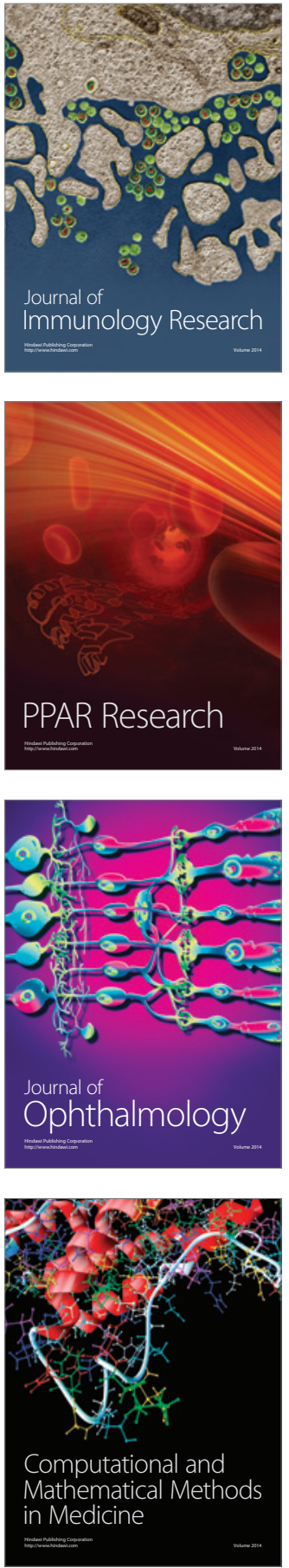

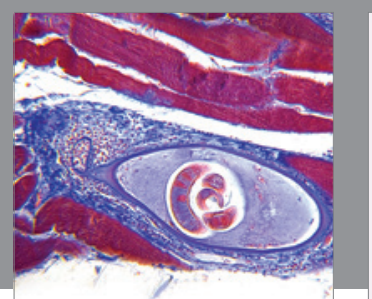

Gastroenterology Research and Practice

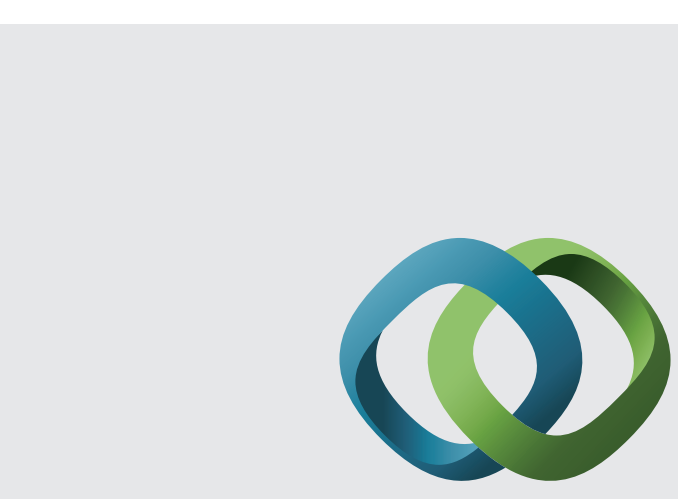

\section{Hindawi}

Submit your manuscripts at

http://www.hindawi.com
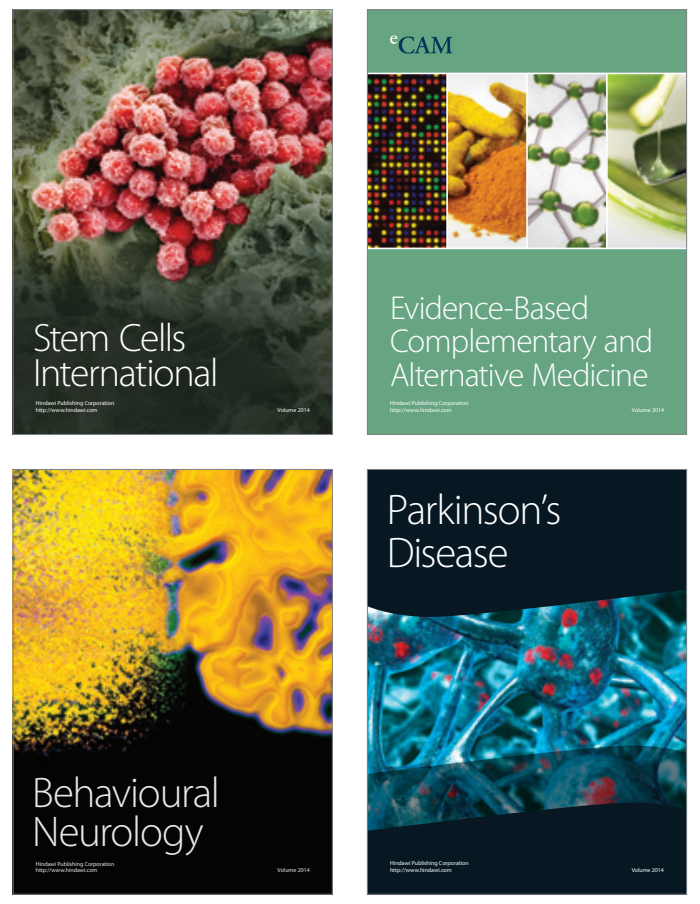
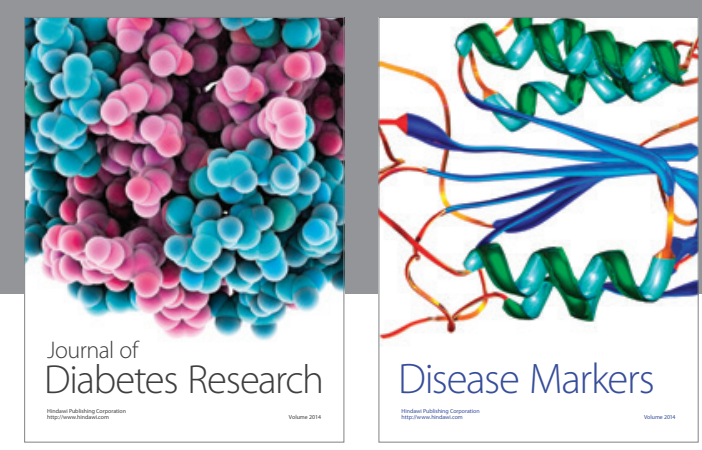

Disease Markers
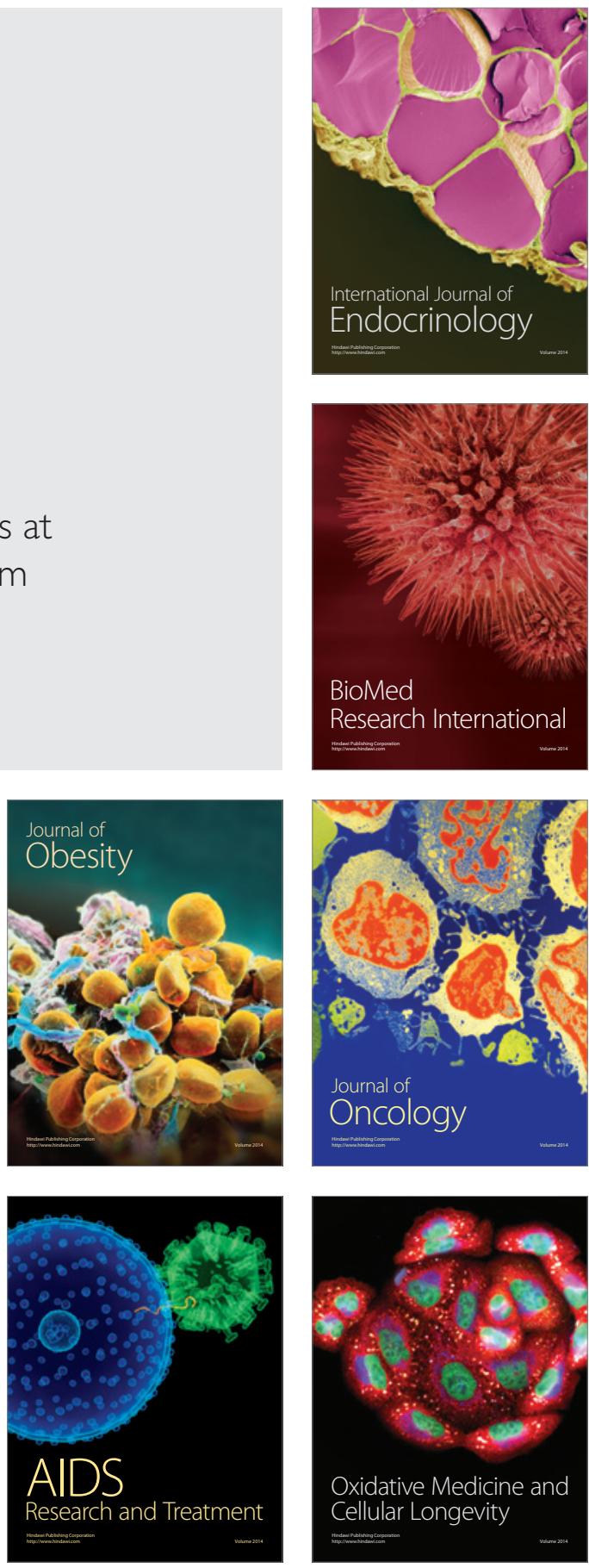